\title{
Probing Protein Surface with a Solvent Mimetic Carbene Coupled to Detection by Mass Spectrometry
}

\author{
Gabriela E. Gómez, ${ }^{1}$ Mariana R. Mundo, ${ }^{1}$ Patricio O. Craig, ${ }^{1,2}$ José M. Delfino ${ }^{1}$ \\ ${ }^{1}$ Departamento de Química Biológica, Facultad de Farmacia y Bioquímica, Universidad de Buenos Aires e Instituto de \\ Química y Fisicoquímica Biológica (IQUIFIB-CONICET), Junín 956, C1113AAD, Buenos Aires, Argentina \\ ${ }^{2}$ University of California, San Diego, CA, USA
}

\begin{abstract}
Much knowledge into protein folding, ligand binding, and complex formation can be derived from the examination of the nature and size of the accessible surface area (SASA) of the polypeptide chain, a key parameter in protein science not directly measurable in an experimental fashion. To this end, an ideal chemical approach should aim at exerting solvent mimicry and achieving minimal selectivity to probe the protein surface regardless of its chemical nature. The choice of the photoreagent diazirine to fulfill these goals arises from its size comparable to water and from being a convenient source of the extremely reactive methylene carbene $\left(: \mathrm{CH}_{2}\right)$. The ensuing methylation depends primarily on the solvent accessibility of the polypeptide chain, turning it into a valuable signal to address experimentally the measurement of SASA in proteins. The superb sensitivity and high resolution of modern mass spectrometry techniques allows us to derive a quantitative signal proportional to the extent of modification (EM) of the sample. Thus, diazirine labeling coupled to electrospray mass spectrometry (ESI-MS) detection can shed light on conformational features of the native as well as non-native states, not easily addressable by other methods. Enzymatic fragmentation of the polypeptide chain at the level of small peptides allows us to locate the covalent tag along the amino acid sequence, therefore enabling the construction of a map of solvent accessibility. Moreover, by subsequent MS/MS analysis of peptides, we demonstrate here the feasibility of attaining amino acid resolution in defining the target sites.
\end{abstract}

Key words: Protein conformation, Diazirine, Accessible surface area, Methylene carbene, Photochemical probe

\section{Introduction}

$\mathrm{P}$ roteins are fundamental building blocks supporting cell structure and most every metabolic reaction. Current efforts in structural biology focus on the underlying basis of

Electronic supplementary material The online version of this article (doi:10.1007/s13361-011-0266-x) contains supplementary material, which is available to authorized users.

Correspondence to: José Delfino; e-mail: delfino@qb.ffyb.uba.ar protein folding, ligand binding, and complex formation. In this regard, several biophysical and biochemical techniques converge to shed light on protein structure and dynamics. Xray crystallography and NMR represent the cornerstones of structural information. In addition, optical methods (circular dichroism, fluorescence, light scattering), chemical techniques (H/D exchange, chemical modification of amino acid side-chains), hydrodynamic properties (size-exclusion chromatography), and molecular biology methods (site-directed mutagenesis, truncations, construction of chimaeras, etc.) have been used to address specific structural aspects of 
proteins. Although individually unable to provide the complete picture, these techniques become very valuable because they can address different structural features and the various time-scales of the folding process [for general references see 1-4].

Inextricably associated with the mechanisms of protein folding or oligomerization is the fact that the solvent accessible surface area (SASA) of the polypeptide chain becomes minimized. A fundamental issue here is the description of the extent and nature of the SASA at each stage of these processes. Nevertheless, despite being a parameter of great importance in protein science [5, 6], there is hardly any experimental method suitable for approaching a direct measurement of SASA. In this regard, parameters such as the heat capacity changes $(\Delta \mathrm{Cp})$ between conformational states [7-10], the cooperativity of a conformational transition as measured by the slope $(\mathrm{m})$ of the curve [11], and the changes in the radius of gyration $(\Delta \mathrm{Rg})$ upon protein folding [12] are thought to be correlated with changes in the accessibility of the whole polypeptide chain, but do not directly yield local information. In a different vein, the profile of chemical reactivity of a given functional group present on an amino acid side chain against a set of reagents of different nature provides a description of the environment around this group, thus making possible to infer local solvent accessibility [13, 14 and references cited therein]. However, by definition, selective chemical modification is limited to a single or a few chemical functionalities, rarely avoiding side reactions and frequently inducing conformational changes. Of widespread application in protein folding $[15,16]$ and protein interactions [17] has been the H/D exchange technique. The exchange rate of the backbone $\mathrm{H}$ amides is influenced by the accessibility to the solvent, the hydrogen bonding state, and the chemical environment. Despite its wide dynamic range, H/D exchange data is intrinsically limited to amide protons belonging to the backbone chain, therefore becoming strongly dependent on secondary structure integrity. This technique addresses the frequency and size of the opening reactions associated with local and global unfolding conformational fluctuations, but does not correlate very well with solvent accessibility. Besides, one main drawback arises from the labile nature of the label. This reversible modification brings about limitations if extensive purification and further analytical processing of the sample is needed to attain local information.

A separate group of footprinting chemical techniques aims at targeting all solvent accessible sites, while concurrently obviating the chemical selectivity as much as possible, thus allowing to map the topography of the folded chain. Prominent among these tools is the use of hydroxy radical $(\cdot \mathrm{OH})$ reactions to explore the structure and interactions involving nucleic acids [18-20] and proteins [21-28]. However, the $\cdot \mathrm{OH}$ species shows some chemical selectivity in its reaction with peptide targets, thus potentially biasing the analysis to particular spots. Moreover, the disruption of the polypeptide chain gives rise to products that are generally stable, but most often radical triggered reactions generate ill-characterized derivatives that complicate the ensuing analytical procedures.

A formally related, but essentially dissimilar approach, relies on the application of electron-deficient species, such as nitrenes or carbenes, for the modification reaction [29-32]. In addition, to achieve the closest geometrical mimicry to the water solvent, the choice of methylene $\left(: \mathrm{CH}_{2}\right)$, the smallest and most reactive member of this family, emerges as a natural candidate $[6,33]$. To serve as a useful labeling reagent, : $\mathrm{CH}_{2}$ has to be generated in situ from a suitable stable precursor. Possibly the best source of methylene is diazirine $\left(\mathrm{CH}_{2} \mathrm{~N}_{2}\right)$, an essentially inert compound unless irradiated (at $\sim 320 \mathrm{~nm}$ ) or heated. Upon irradiation diazirine generates the reactive $: \mathrm{CH}_{2}$ species, which inserts readily into any $\mathrm{X}-\mathrm{H}$ bond $(\mathrm{X}: \mathrm{C}, \mathrm{O}, \mathrm{N}$, or $\mathrm{S})$, yielding defined and stable methylated products minimally different in their physicochemical character from the unreacted counterparts $[34,35]$ (Scheme 1). Due to its extremely short half-life, : $\mathrm{CH}_{2}$ will react with target groups belonging to its immediate molecular cage regardless of their chemical nature. At present the main proof of the indiscriminate reactivity of : $\mathrm{CH}_{2}$ with proteins comes from the uniformity of the labeling yield observed in the unfolded state of bovine $\alpha$-lactalbumin $[33,36]$ and Bacillus licheniformis $\beta$-lactamase [37] at the level of tryptic and CNBr-derived peptides, in spite of differences in the amino acid composition of these peptides. This behavior and the minimal size of the precursor molecule represent key features of this experimental approach to probing the surface theoretically defined as SASA.

Unlike H/D exchange, labeling with diazirine would target side chains as well as backbone exposure. Most importantly, the modification with $: \mathrm{CH}_{2}$ involves the formation of strong covalent bonds, giving rise to stable products that broaden the range of subsequent analytical procedures $[6,33]$. On the other hand, the extent of protein or water labeling results proportional to their relative abundance in the solution [33]. Despite the fact that most

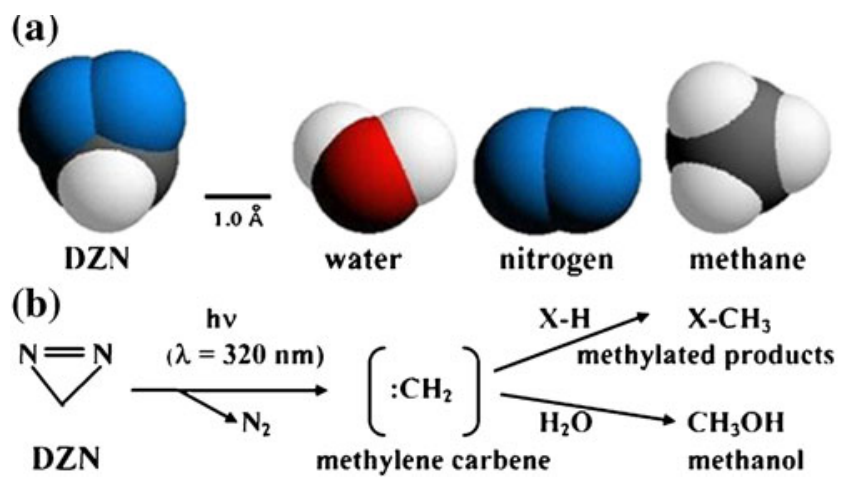

Scheme 1. (a) Comparative size of diazirine (DZN) with respect to water and other small molecules. (b) Photolysis reactions of diazirine 
of diazirine reacts with the aqueous solvent (yielding methanol), this does not compromise the adequate detection by mass spectrometry (MS) of labeled proteins. The build-up of methanol at low millimolar concentration poses no disturbance to protein conformation. Indeed, in previous work, samples subjected to diazirine reagent after irradiation were controlled by the very sensitive standard given by farand near-UV CD spectroscopy, showing identical spectra to non-treated samples [33, 37]. This evidence rules out any putative change to conformation as a result of the presence of the diazirine reagent and the irradiation conditions. An added advantage of the technique is the fact that the rate of diazirine photolysis in aqueous solution remains invariable under diverse conditions of $\mathrm{pH}$, ionic strength, or concentration of chaotropes commonly used [33, 36, 37].

In previous work of our laboratory, the tritiated form of diazirine $\left({ }^{3} \mathrm{H}\right.$-diazirine) allowed us to accurately estimate the global extent of modification of the protein, as well as to identify the site of labeling along the polypeptide. ${ }^{3} \mathrm{H}-$ diazirine has been successfully employed to address unfolding transitions of single-domain proteins $[33,36,37]$ and the contact surface of an antigen-antibody complex [38]. Other researchers took advantage of diazirine labeling to identify an alternative conformation of replication protein A [39]. Despite the proven usefulness of the tritiated version of the reagent, obstacles remained in the way to a generalized use of this methodology. Among these are the cumbersome handling and the expense of radiotracers, and the large amounts of protein needed for the analysis, as a consequence of the low specific radioactivity of the precursor molecules. On the other hand, the advent of extremely sensitive and high resolution MS techniques has already been applied to investigate conformation, dynamics, and interactions involving macromolecules $[40,41]$. In this context, we introduce an alternative (nonradioactive) signal useful to estimate the methylation extent of samples. Here we substantiate this analysis for archetypal proteins, demonstrating the value of the technique to assess conformational states. Moreover, we advance into the ability of the method to pinpoint sites along the chain, revealing key structural features of the folded form.

\section{Materials and Methods}

Formaldehyde (37\%, wt/vol) was from E. Merck (Darmstadt, Germany); formamide, urea, human ubiquitin, bovine $\alpha$-lactalbumin, hen egg white lysozyme, bovine brain calmodulin, and subtilisin A from Bacillus sp were from Sigma Chemical Co. (St. Louis, MO, USA). Bacillus licheniformis $\beta$-lactamase was a gift from Dr. Daniela Ureta (Instituto de Química y Fisicoquímica Biológica, UBA-CONICET, Buenos Aires, Argentina). TPCK trypsin and $\alpha$-chymotrypsin from bovine pancreas were from Worthington Biochemical Corporation (Lakewood, NJ, USA). All proteins were used without further purification. Urea was recrystallized from ethanol before use. Acetonitrile from E. Merck
(Darmstadt, Germany) and trifluoroacetic acid from Riedel de Haën (Seelze-Hannover, Germany) were of HPLC grade. All other reagents and chemicals used were of analytical grade.

\section{General Procedures}

Protein concentration was determined by their UV absorption on a Jasco 7850 spectrophotometer, using the following extinction coefficients at $280 \mathrm{~nm}: \mathrm{E}_{1 \%}$ ubiquitin $=1.49$ [42], $\mathrm{E}_{1 \%} \alpha_{\text {-lactalbumin }}=20.1$ [43], $\mathrm{E}_{1 \% \text { lysozyme }}=26.5$ [44], $\mathrm{E}_{1 \% \text { subtilisin }}=$ 8.6 [45], $\mathrm{E}_{1 \% \text { p-lactamase }}=7.68$ [46], $\mathrm{E}_{1 \% \text { trypsin }}=14.4$ [47] and $\mathrm{E}_{1 \%}$ chymotrypsin $=20.8$ [48]. For calmodulin, a molar extinction coefficient at $277 \mathrm{~nm}$ of $3300 \mathrm{M}^{-1} \mathrm{~cm}^{-1}$ was used [49]. To avoid auto-digestion, trypsin, and chymotrypsin were dissolved in $40 \mathrm{mM}$ sodium acetate buffer at $\mathrm{pH} 4$. The remaining proteins were solubilized in $20 \mathrm{mM}$ phosphates buffer at $\mathrm{pH}$ 7.4. Except for the experiment described in Figure 2, where $6 \mathrm{M}$ guanidinium chloride $(\mathrm{GuHCl})$ was used, all the other buffers were added with urea up to $8 \mathrm{M}$ to stabilize proteins in their unfolded states. All buffers were degassed under vacuum and flushed with nitrogen gas.

\section{Circular Dichroism Spectroscopy}

Spectra were recorded on a Jasco J-810 spectropolarimeter, using quartz cylindrical cuvettes of 1 or $10 \mathrm{~mm}$ path lengths for the far (200-250 nm) or near (250-310 nm) UV regions, respectively. In every case, to enhance the signal-to-noise ratio, five consecutive spectra were recorded and averaged. Data were converted to molar ellipticity $[\theta]_{\mathrm{M}}$ (in units of deg $\mathrm{cm}^{2} \mathrm{dmol}^{-1}$ ) using a mean residue weight value for $\alpha$-lactalbumin of $115.5 \mathrm{~g} / \mathrm{mol}$.

\section{Fluorescence Spectroscopy}

Measurements were carried out with an Aminco Bowman Series II spectrofluorometer, using a cuvette of $0.4 \mathrm{~cm}$ path length. For intrinsic (tryptophan) fluorescence measurements, we used excitation and emission bandwidths of $4 \mathrm{~nm}$, an excitation wavelength of $295 \mathrm{~nm}$ to selectively excite $\mathrm{W}$ residues present in the protein, and the emission was collected in the range $310-450 \mathrm{~nm}$.

\section{Synthesis of Diazirine}

Diazirine was synthesized in our laboratory by following the method described by Craig et al. [33]. Briefly, the procedure is a modification of the method described by Ohme and Schmitz [50]. This involves four steps starting from (1) vacuum concentration of $37 \%(\mathrm{wt} / \mathrm{vol})$ formaldehyde to yield paraformaldehyde, (2) condensation of this solid with formamide at $125^{\circ} \mathrm{C}$ for $1-2 \mathrm{~d}$, (3) hydrolysis of the product methylene-bis-formamide with $50 \%$ (wt/wt) aqueous $\mathrm{H}_{2} \mathrm{SO}_{4}$, and (4) oxidation of the cation of the resulting salt, methylene diammonium sulfate, with aqueous $1 \mathrm{~N} \mathrm{NaClO} /$ $0.9 \mathrm{M} \mathrm{NaOH}$. 
Diazirine gas was purified from remaining oxidizing materials that could potentially be deleterious to the polypeptide, and from traces of the synthetic intermediate diaziridine by successive passage through sodium bisulfite and citric acid columns, respectively [6]. Diazirine concentration in aqueous solution was estimated by measuring the absorbance of the dissolved gas at $320 \mathrm{~nm}$ using an extinction coefficient of $\varepsilon_{320}=180 \mathrm{M}^{-1} \mathrm{~cm}^{-1}$ [33]. Typically, the initial diazirine concentration falls in the range $4.5-5.5 \mathrm{mM}$.

Although the possibility of flash burn explosions of diazirine has been reported [6], this is only of some concern if handling the reagent in the condensed state at high concentration. However, this is not an issue at the dilute aqueous solutions commonly used in our experiments.

\section{Design of a Photolysis Device for the Chemical Modification of Proteins with Diazirine}

To enhance and control the extent of modification of proteins with diazirine (Scheme 1), we developed a device that takes advantage of the continuous input and photolysis of the gas into the sample (Figure 1).

Diazirine is generated in a $100 \mathrm{~mL}$ glass syringe where the precursor salt (methylene diammonium sulfate) is mixed

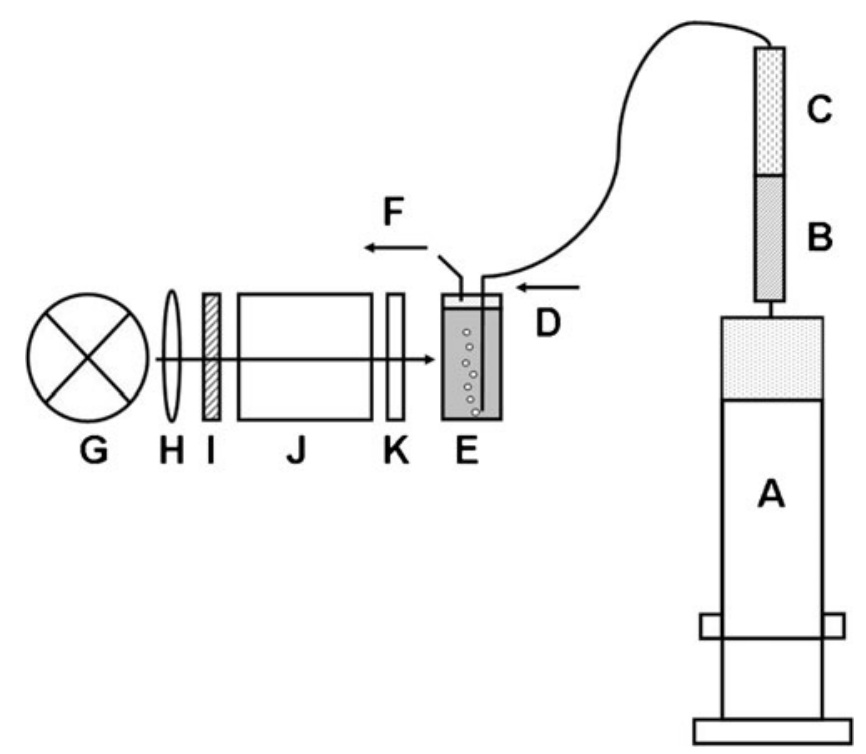

Figure 1. Photolysis device for the chemical modification of proteins with diazirine. The different parts of the system are indicated as follows. Diazirine gas contained in the glass syringe (A); on-line sodium bisulfite (B)/citric acid (C) filter; diazirine inflow into the stirred protein solution (D); sample placed into a thermostatted quartz cuvette (E); outlet tube for gas outflow (F); UV light source (G); condenser lens (H); automated shutter (I); water IR filter (J); cut-off filter $(\lambda<$ $300 \mathrm{~nm})(\mathbf{K})$. For additional details, see Materials and Methods with an alkaline solution of sodium hypochlorite. An automated syringe drive unit controls gas flow (typically in the range $0.8-1.0 \mathrm{~mL} / \mathrm{min}$ ). An on-line combined citric acid/ sodium bisulfite filter column traps traces of impurities. Diazirine is bubbled through a stainless steel tube into a stirred protein solution $(1-4 \mathrm{~mL})$ to which octanol $(1 \mu \mathrm{L})$ was added as an antifoaming agent. This solution is placed into a capped quartz cuvette $(1 \mathrm{~cm}$ width $\times 1 \mathrm{~cm}$ depth $\times$ $4.8 \mathrm{~cm}$ height) thermostatted at room temperature by flowing air. If necessary, an outlet tube can be connected to a receiving syringe to exert controllable back pressure on the sample. All joints were sealed to avoid any loss of diazirine gas.

The photolysis setup (Oriel) consists of a $1000 \mathrm{~W} \mathrm{Hg} / \mathrm{Xe}$ arc source (Oriel code 6295) equipped with a back reflector, F/1 condenser lenses, water IR filter, automated shutter to control the time of exposure, and a cut-off filter (Oriel code 59044, $\lambda<300 \mathrm{~nm})$ to prevent damage to the protein chromophores. All elements are mounted on an optical bench. This arrangement allows the beam to be efficiently focused into the sample compartment, thus conveniently reducing the lifetime of photolysis.

The chemical modification reaction is normally carried out in the steady-state regime because under this condition the yield of product is maximized while minimizing the light exposure of the sample. In practice, the steady-state concentration of diazirine can be reached in our device by setting the input rate to match the photonic flux on the sample.

The evolution of diazirine concentration in solution (C) is described by the differential Equation (1), where the second term represents the diazirine increment due to the input of gas into the sample and its partition into the aqueous phase, and the third term accounts for the diazirine decrement as a consequence of its photolysis and loss through the outlet tube.

$$
\frac{d C}{d t}-\left(\frac{C_{s} F_{i}}{K V_{g}+V}\right)+\left(\frac{K F_{o}+k V}{K V_{g}+V}\right) C=0
$$

where $\mathrm{C}_{\mathrm{s}}$ is the concentration of diazirine gas in the syringe reservoir; $F_{i}$ and $F_{o}$ are the input and output gas flows, respectively; $\mathrm{K}$ is the gas-liquid partition coefficient of diazirine; $\mathrm{k}$ is the pseudo first-order photolysis rate; and $\mathrm{V}$ and $\mathrm{V}_{\mathrm{g}}$ are the volumes of the liquid and gas phases in the cuvette, respectively. By definition, the condition for steadystate means the following: $\frac{d C}{d t}=0$. In addition, in a typical experiment, the loss of diazirine through the outlet tube is much lower that its loss due to photolysis, i.e., $K F_{o} C \ll k V C$. Thus, Equation (1) becomes the following:

$$
\frac{C_{S} F_{i}}{V} \approx C_{0} k
$$

where $\mathrm{C}_{0}$ is the steady-state concentration of diazirine.

Indeed, as one can monitor the time evolution of diazirine concentration in solution by UV absorption at $320 \mathrm{~nm}$, the 
ratio $\frac{C_{s} F_{i}}{V}$ (diazirine concentration in $\mathrm{AU} \cdot \mathrm{min}^{-1}$ ) can be determined by measuring the initial slope of the dissolution of diazirine $(\mathrm{m})$ into the sample in the absence of photolysis $(k=0)$. On the other hand, $\mathrm{k}$ can also be independently determined in a closed system $\left(F_{i}=F_{o}=0\right)$. Thus:

$$
C_{0} \approx \frac{m}{k}
$$

Equation (3) allows one to estimate $\mathrm{C}_{0}$ a priori, so that one can set the initial diazirine concentration in the sample as close as possible to that value. In fact, this prediction was checked against the actual steady-state diazirine concentration measured in a given experiment (data not shown).

Equation (4) describes the yield of methylated products (P) as a function of the time of photolysis ( $\mathrm{t}$ ) and diazirine concentration in the liquid phase $(\mathrm{C})$. Indeed, $\int C d t$ is the load of diazirine reagent in the sample estimated for each experiment as the area under the curve in a plot of $\mathrm{C}$ (measured as $\mathrm{A}_{320}$ ) versus time.

$$
P=k \int_{0}^{t} C d t
$$

Under steady-state conditions the expression becomes the following:

$$
P=k C_{0} t
$$

A meaningful comparison among results from independent experiments requires checking accurately the load of diazirine reagent.

\section{Definition of the New Metric EM (Extent of Modification with Methylene) Derived from MS Data}

In this work, we sought to obtain from each MS spectrum a quantitative measure that reflects the extent of reaction of a protein with methylene carbene. Although ESI-MS fulfills ideally the aim of determining the molecular mass of a species from the position of the peak, there is scant information as to whether peak intensities can be used to derive the composition of a given sample. The extent of modification (EM) can be approximated by the following expression:

$$
E M=\sum_{i=0}^{i=n} i I_{i} / \sum_{i=0}^{i=n} I_{i}
$$

where $I_{i}$ represents the intensity of peak $i$, i being the index that indicates the number of methylene groups incorporated per protein molecule. To calculate EM, it is essential to have available the collection of all species present (including the unmodified form) in the same spectrum. On the other hand, the chemical alteration of the polypeptide introduced by the diazirine reaction (methylation) allows us to expect minimal differences in regard to the ionization properties of the molecular species present in the sample. Thus, EM can be estimated by the following expression:

$$
E M \approx(\langle M\rangle-M) / P S
$$

where $\mathrm{M}$ is the mass of the unmodified protein, $<\mathrm{M}>$ is the intensity averaged molecular mass and PS is the average peak separation, as defined below:

$$
\begin{gathered}
\langle M\rangle=\sum_{i=0}^{i=n} I_{i} X_{i} / \sum_{i=0}^{i=n} I_{i} \\
P S=(1 / n) \sum_{i=0}^{i=n}\left(X_{i}-X_{0}\right) / i
\end{gathered}
$$

In Equations (8) and (9), $I_{i}$ represents the intensity of peak $\mathrm{i}$ approximated as a Gaussian function centered at molecular mass $X_{i}$, and $X_{0}(=M)$ corresponds to the molecular mass of the unmodified species. To estimate these parameters, a minimal set of Gaussian functions plus a linear baseline were fit to the experimental spectra. The PS parameter and its associated error indicate the extent to which and how evenly peaks are spaced in the mass domain.

Finally, to draw meaningful comparisons among results from independent experiments, EM values, calculated from individual spectra and expressed as moles of : $\mathrm{CH}_{2}$ incorporated per mole of protein, should be normalized by the load of diazirine reagent.

\section{Analysis of Protein Samples by RP-HPLC Followed by ESI-MS}

Diazirine reacted and control protein samples were run on a C8 column (Vydac 208TP5105, 50×1 mm) developed with two successive linear gradients of solvent B (2\% water, $2 \%$ acetic acid, 96\% acetonitrile) in solvent A (2\% acetonitrile, $2 \%$ acetic acid, $96 \%$ water): $0-5 \% \mathrm{~B}$ in $2 \mathrm{~min}, 5 \%-100 \% \mathrm{~B}$ in $1.5 \mathrm{~min}$, followed by $100 \% \mathrm{~B}$ for $5 \mathrm{~min}$, at a flow rate of $40 \mu \mathrm{L} / \mathrm{min}$. The column is attached to a Thermo Surveyor HPLC system coupled to an electrospray mass spectrometer (ESI-MS; Thermo Finnigan LCQ duo, equipped with an ion trap mass analyzer). All ESI-MS spectra shown have been deconvoluted to the zero-charge domain with the software Pro Mass, using a standard parameter set: average mass type, mass tolerance $0.02 \%$, minimum tolerance $2 \mathrm{Da}$, relative impurity threshold $30 \%$. Input $\mathrm{m} / \mathrm{z}$ range $300-2000$, adduct 
ion mass 1.0079 , baseline removal set at 0.8 , peak width 1 , merge width 0.3 , minimum score 2 , normalize scores 1 , comprehensive deconvolution set to on, smooth width 5 , number of smoothes 2 , noise threshold set at $0.1 \%$ relative intensity.

\section{Reduction, Carbamido Methylation, and Tryptic Digestion of Diazirine Labeled Lysozyme}

Photolabeled and control lysozyme samples intended for further peptide analysis were reduced with DTT and blocked with iodoacetamide according to Waxdal et al. [51] with the modifications described by Craig et al. [33]. Complete enzymatic digestion of these carbamido methylated samples with TPCK trypsin was achieved in $\mathrm{NH}_{4} \mathrm{HCO}_{3}$ buffer (0.1 $\mathrm{M}$ at $\mathrm{pH} 8.0$ ), after $12-24 \mathrm{~h}$ at $37{ }^{\circ} \mathrm{C}$ using a $2 \%$ (wt/ wt) enzyme:substrate ratio. The mixture was sampled onto a C18 column (Vydac 214TP54, 250×4.6 mm) developed with a linear gradient of acetonitrile in water added with $0.05 \%$ TFA $(0-100 \%$ in $50 \mathrm{~min})$ at a flow rate of $1 \mathrm{~mL} / \mathrm{min}$. Elution was monitored by UV absorption at $215 \mathrm{~nm}$. The peptide fraction eluting between $10 \%$ and $60 \%$ were pooled together and freeze-dried.

\section{MALDI MS of Proteolytic Digests}

A droplet of each peptide sample $(0.5 \mu \mathrm{L}, \sim 1 \mathrm{pmol} / \mu \mathrm{L})$ dissolved in $60 \%$ acetonitrile, $0.1 \%$ TFA in water was allowed to dry. Then, a saturated matrix solution of $\alpha$ cyano4-hydroxycinnamic acid $(0.5 \mu \mathrm{L}, \sim 10 \mathrm{mg} / \mathrm{mL})$ in $70 \%$ acetonitrile, $0.1 \%$ TFA in water was added onto the spot and also allowed to dry. Samples were ablated with a pulsed Nd:YAG laser $(355 \mathrm{~nm})$ at a nominal power of 3400 arbitrary units. All MALDI spectra were recorded on an Applied Biosystems MALDI TOF/TOF 4800 Plus mass spectrometer operating in the reflector mode, by applying an accelerating voltage of $20 \mathrm{kV}$ and the delayed extraction time set to $450 \mathrm{~ns}$. Each spectrum represents the average of 500 laser shots and is externally calibrated.

Unambiguous identification of tryptic peptides was achieved after MALDI-MS/MS and post source decay (PSD) fragmentation over selected ions at a laser power of 4400 arbitrary units.

\section{RP-HPLC Separation of Peptide Samples Followed by ESI-MS}

Peptide samples were run on a C18 column (Phenomenex Jupiter Proteo, $150 \times 1 \mathrm{~mm}$ or Vydac 218TP5105, 50× $1 \mathrm{~mm}$ ) developed with a linear gradient of solvent $\mathrm{B}$ ( $80 \%$ acetonitrile, $0.1 \%$ formic acid in water) in solvent A ( $0.1 \%$ formic acid in water): $2-100 \% \mathrm{~B}$ in $90 \mathrm{~min}$. The eluate was interfaced to the ESI mass spectrometer described above. The detector was operated in the datadependent mode to automatically switch between MS and
MS/MS acquisition. MS/MS spectra were generated for preselected ionic species by collisionally induced dissociation (CID) with helium gas in the ion trap at a collision energy setting of $35 \%$ (arbitrary units). Prediction of the fragmentation pattern was achieved with an ad hoc macro built over an Excel spreadsheet that takes into account the appearance of methylated species. Product ion series was confirmed with GPMAW 8.10 (2008; Lighthouse data, Odense, Denmark) kindly provided by Dr. Ulf Hellman (Ludwig Institute for Cancer Research, Uppsala, Sweden). All MS analyses were carried out at our local protein facility (LANAIS-PRO, UBA-CONICET).

\section{Molecular Modeling}

Calculations of the solvent accessible surface area (SASA) of lysozyme based on high-resolution crystallographic structures (pdb codes 1hew and 1lzt) were carried out with Surface Racer [52] using van der Waals atomic radii according to the parameter set defined by Richards [5] with a choice of $1.4 \AA$ probe radius for the water molecule.

\section{Results and Discussion}

\section{Diazirine Reacted Proteins Reveal a Characteristic Mass Signature of Methylated Products}

An optimal detection of methylated products by ESI-MS put forward the challenge of developing a suitable device for the chemical modification of proteins with diazirine. The apparatus takes advantage of the continuous input and simultaneous photolysis of diazirine gas into the protein sample in solution. The possibility of varying the load of the photoreagent in the sample allows one to tune up the yield of the photoreaction at will (see Materials and Methods). At the level of a full-length protein (hen egg white lysozyme), ESIMS allowed us to distinguish methylated products as individual $\mathrm{M}+\mathrm{i} \cdot 14$ peaks, where $\mathrm{i}$ corresponds to the number of methylene groups incorporated per protein molecule (Figure S1). The overall intensity profile of peaks is consistent with a random modification of the polypeptide chain [6]. Typically, the spectrum of a diazirine modified sample of lysozyme reveals the peak corresponding to the unmodified species $(M=14305 \mathrm{Da})$ followed by a pattern of methylated products from which a reliable quantitative signal indicating the extent of modification (EM) with methylene can be derived. The new metric EM is expressed in units of moles of : $\mathrm{CH}_{2}$ incorporated per mole of protein, normalized at a given concentration of diazirine (see Materials and Methods). Figure S1 also shows that no detectable concurrent photolytic damage takes place, because the spectrum of a control sample irradiated without diazirine results indistinguishable from that of an untreated sample. 


\section{The EM Signal Depends Linearly on the Load of Diazirine}

To validate the use of EM as an estimate of the extent of modification, we investigated the dependence of this parameter with the load of the reagent, the latter being measured as the instantaneous concentration of diazirine reagent in the sample times the irradiation period $\left(\int\right.$ [diazirine] dt). This point was tested in a set of lysozyme samples treated with diazirine under steady-state conditions at different times of photolysis (Figure S2). EM follows a linear dependence with the load of the reagent in the range assayed. The fitted line shows that at null diazirine load, the extrapolated value of EM does not differ significantly from zero. These results demonstrate that the linear behavior observed before by using the tritiated version of the diazirine probe $[33,37]$ holds true for an extended range well above that explored with the radioactive reagent. Moreover, the linear regime warrants the possibility of adequately managing the conditions for the chemical modification, so that a straightforward comparison among samples can be drawn. In this fashion, conformational states could be optimally discriminated (see below).

\section{EM Proves to be Sensitive to Conformational Change}

Figure 2 illustrates the ESI mass spectra of two lysozyme samples, where the native or the unfolded states of this protein are populated, labeled under identical reaction conditions, i.e., with the same load of diazirine. From the comparison, it becomes immediately obvious that a large shift occurs toward heavier mass peaks in the unfolded form of protein. Thus, the EM parameter, which is able to quantify this difference, responds to conformational change. Alternatively, diazirine modified protein samples were also assayed by MALDI-MS (data not shown). Here, the lower resolution of MALDI-MS for entities of this molecular weight $(14.3 \mathrm{kDa})$ precludes the identification of individual methylated species. Samples of bovine $\alpha$-lactalbumin labeled in its native or unfolded states were assayed in this fashion. Although one can attempt to derive a useful signal from the featureless shape of the molecular weight distribution envelope, in this case different conformational states of the protein can only be assessed at a semiquantitative level. In this fashion, EM calculated from ESI-MS emerges as the ideal choice for the analysis of full proteins.

To test the general nature of our observations beyond the two cases above, we labeled several globular proteins in their native or unfolded states (Table 1). For all the proteins assayed, the EM parameter is higher in the latter case, a fact consistent with the expected SASA increment occurring upon unfolding. In addition, as the molecular weight (and consequently the SASA) of the protein increases, a correspondingly higher EM is also observed. Remarkably, lysozyme and $\alpha$-lactalbumin, two sequentially unrelated but conformationally similar proteins of about the same molecular weight (14.3 kDa), show comparable EM values in the native state. These results emphasize the nonspecific nature of the diazirine labeling phenomenon, in agreement with experiments run with the tritiated version of the reagent [36].

One should note that under the selected experimental conditions, the EM values are indeed low in all cases. Far from constituting a disadvantage, this is precisely the aim sought out here: to modify the protein at just the level needed to extract a meaningful and accurate signal, thus avoiding the risk of introducing any potential perturbation due to the very modification phenomenon. Indeed, a

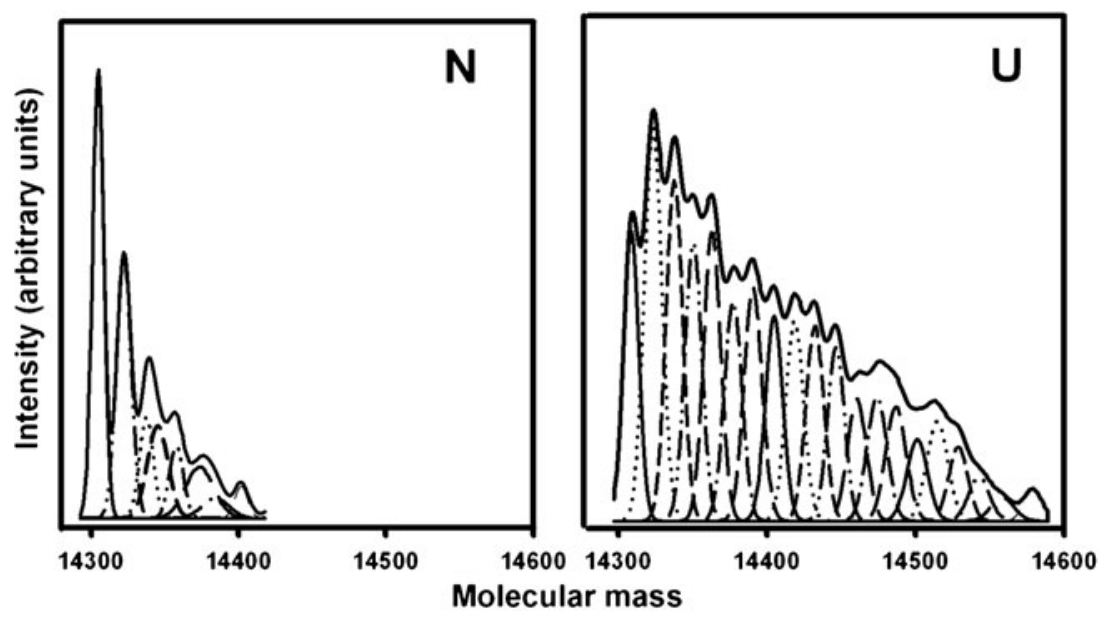

Figure 2. Labeling with diazirine is sensitive to protein conformation. Deconvoluted ESI-MS spectra of lysozyme samples $(2 \mathrm{mg} / \mathrm{mL})$ labeled in the native $(\mathrm{N})$ or unfolded $(\mathrm{U}, 6 \mathrm{M} \mathrm{GuHCl})$ states. The conditions for modification were: diazirine inflow rate of $0.8 \mathrm{~mL} / \mathrm{min}$, input plus photolysis phase lasting $3.5 \mathrm{~min}$, steady-state diazirine concentration $\left(\mathrm{C}_{0}\right) \sim 0.8 \mathrm{AU}$, for details see Materials and Methods. A function (solid line) representing the sum of a set of Gaussian components (dashed lines) was fitted to the experimental data (solid circles). Average peak separation (PS) was $14.2 \pm 1.3$ and $13.9 \pm 0.3 \mathrm{u}$ for panels $\mathrm{N}$ and $\mathrm{U}$, respectively. No significant data above the threshold value (set as zero in the graph) exist outside the range shown 
Table 1. Extent of Modification (EM) of Proteins Labeled in the Native (N) or Unfolded (U, 8 M urea) States

\begin{tabular}{lcc}
\hline \multirow{2}{*}{ Protein $^{\mathrm{a}}$} & \multicolumn{2}{c}{$\mathrm{EM}\left(\mathrm{mol}: \mathrm{CH}_{2} / \mathrm{mol} \text { of protein }\right)^{\mathrm{b}}$} \\
\cline { 2 - 3 } \multicolumn{1}{c}{ Ubiquitin } & $\mathrm{N}$ & $\mathrm{U}$ \\
a-Lactalbumin & $0.15 \pm 0.03$ & $0.27 \pm 0.05$ \\
Lysozyme & $0.99 \pm 0.20$ & $2.77 \pm 0.58$ \\
Calmodulin & $1.00 \pm 0.20$ & $1.62 \pm 0.45$ \\
Trypsin & $0.94 \pm 0.20$ & $1.21 \pm 0.30$ \\
Chymotrypsin & $1.13 \pm 0.22$ & $2.95 \pm 0.58$ \\
Subtilisin & $2.00 \pm 0.38$ & $2.44 \pm 0.50$ \\
$\beta$-Lactamase & $1.86 \pm 0.38$ & $2.08 \pm 0.50$ \\
\hline
\end{tabular}

${ }^{\mathrm{a} U b i q u i t i n}=$ human ubiquitin; $\alpha$-lactalbumin=bovine $\alpha$-lactalbumin; lysozyme=hen egg white lysozyme; calmodulin=bovine brain calmodulin; trypsin=trypsin from bovine pancreas; chymotrypsin $=\alpha$-chymotrypsin from bovine pancreas; subtilisin $=$ subtilisin A from Bacillus $s p ; \beta$-lactamase $=\beta$ lactamase from Bacillus licheniformis. Molecular weights assayed span the range $8.5 \mathrm{kDa}$ (ubiquitin)-29.3 $\mathrm{kDa}$ ( $\beta$-lactamase). In all cases, protein concentration was $1-2 \mathrm{mg} / \mathrm{mL}$. The reaction conditions for modification were as follows: diazirine inflow rate of $1.0 \mathrm{~mL} / \mathrm{min}$, input plus photolysis phase lasting $2 \mathrm{~min}$, steady-state diazirine concentration $\left(\mathrm{C}_{0}\right) \sim 0.8 \mathrm{AU}$. For details see Materials and Methods.

${ }^{\mathrm{b}}$ All numbers shown represent EM values, as defined by Equation (6), normalized by the load of photoreagent expressed in AU.min units.

"sparse" methylation event becomes a desirable goal because it minimizes the chance of introducing unwanted conformational change. Given that the unspecific labeling is linearly dependent on the accessible area of the protein or peptides [36] and reagent concentration [33, 37], (Figure S2 in the Electronic Supplementary Material), any change in the labeling yield, as quantified by the EM value, at the same reagent concentration will be attributed to a change in the accessibility of the polypeptide occurring concomitant with the conformational transition. Overall, EM emerges as a straightforward measurement derived from ESI-MS spectra, useful for analyzing protein conformation from the perspective of the solvent exposure.

In due course, we proceeded to analyze the path of a conformational transition by this technique (Figure 3). The urea-induced conformational shift experienced by $\alpha$-lactalbumin was monitored by following the EM signal alongside known conformational probes such as circular dichroism (in both far and near ultraviolet regions) and the fluorescence emission of tryptophan. Indeed, EM shows a sharp step toward a higher value at a urea concentration close to those revealed by the other spectroscopic probes, thus adding a new dimension to describe a protein cooperative transition.

\section{Identification of Methylene Carbene Labeled Sites by $M S$}

After having assessed the ability of the methylene carbene labeling approach tied to MS detection to probe protein conformation, the need arises to address the issue of identifying labeled sites along the polypeptide chain. Thus, the well-known lysozyme model system proved to be suitable to explore the full scope of the technique. To this

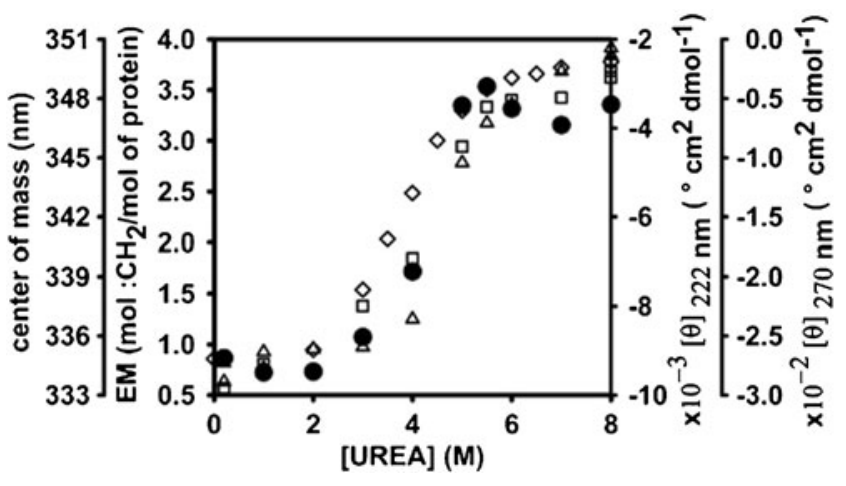

Figure 3. A protein conformational transition monitored by diazirine labeling. The urea-induced equilibrium unfolding of a-lactalbumin $(1 \mathrm{mg} / \mathrm{mL}$ ) was probed by this technique (solid circles), as well as by circular dichroism in both far (open triangles) and near (open squares) ultraviolet regions, and fluorescence emission of tryptophan (open diamonds). The conditions for modification were: diazirine inflow rate of $0.8 \mathrm{~mL} / \mathrm{min}$, input plus photolysis phase lasting $3.5 \mathrm{~min}$, steady-state diazirine concentration $\left(\mathrm{C}_{0}\right) \sim 0.8 \mathrm{AU}$

end, diazirine modified samples of this protein in the native or unfolded states were analyzed by MALDI-MS after reduction, carbamido methylation, and digestion with trypsin. Indeed, the ability to detect modified species at the level of short peptides is illustrated in Figure 4, where peptide 3445 was chosen as an example. Next, as it emerges from the comparison of Figure 4a, b and Figure 4c, d, methylated species derived from this peptide occur exclusively for diazirine treated samples of lysozyme. To achieve this level of detection, the experimental conditions for the modification of the full-length protein $(1.0 \mathrm{~mL} / \mathrm{min}$ diazirine inflow rate, 40 min input + photolysis phase, $\mathrm{C}_{0} \sim 0.9 \mathrm{AU}$ ) had to be more exhaustive than those used before (see Table 1). Nevertheless, the fact that monomethylated species appear in the spectra as the only products is far from paradoxical. The explanation arises from the randomness inherent to the methylene insertion phenomenon, obeying a simple Poissonlike behavior. In this fashion, the expectation holds that only monomethylated species should show up for a tryptic peptide of average length. This modification regime is indeed advantageous because it allows site-specific detection, whereas avoiding the drawbacks intrinsic to an excessive extent of reaction, e.g., the occurrence of conformational change due to the cumulative effect of modification and/or the increasingly higher probability of expecting altered MS behavior in heavily modified products. From the analytical standpoint, measuring the relative proportion of monomethylated species with respect to the unmodified counterpart provides enough information to calculate EM. Thus, Equation (6) becomes $E M \approx I_{1} /\left(I_{0}+I_{1}\right)$. In this fashion, the EM values estimated for tryptic peptides identified by MALDI-TOF mass analysis derived from diazirine labeled lysozyme in the native and unfolded states are shown in Table 2. All tabulated peptides were unambiguously identified by MALDI-MS and ESI-MS/ 


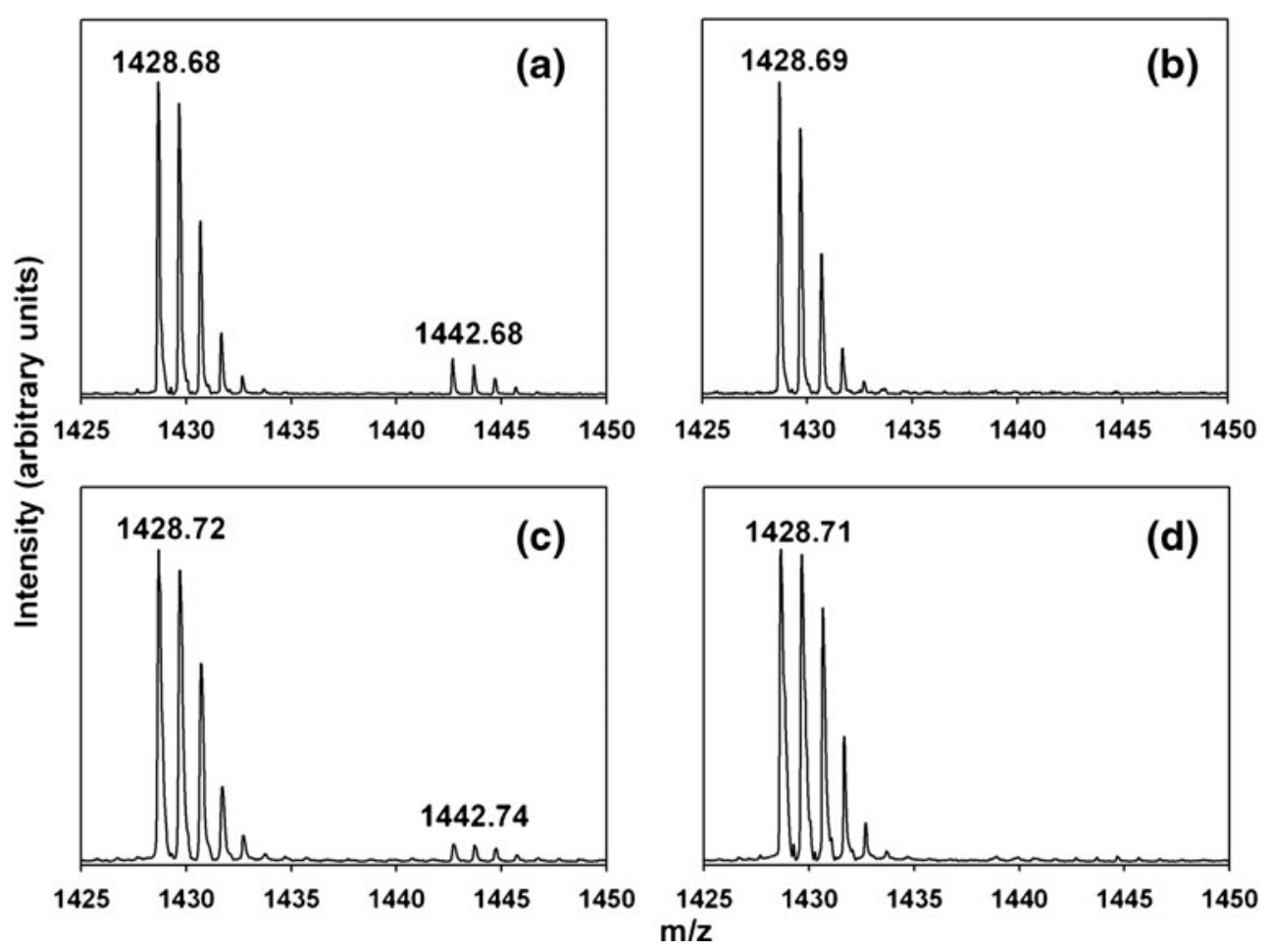

Figure 4. MALDI-MS spectra of peptide $\mathrm{F}_{34} \mathrm{ESNFNTQATNR}_{45}$ derived from lysozyme. Labeling conditions are those described in Table 2. (a) and (c) correspond to samples derived from lysozyme labeled with diazirine in the native or unfolded states, respectively. (b) and (d) show control samples (run in the absence of diazirine and irradiated for the same period) derived from lysozyme in the native or unfolded states, respectively

MS. This collection represents those peptides for which both the unmodified and the monomethylated species could be properly identified and quantitated. Long peptides such as 74-96 $(\mathrm{m} / \mathrm{z} 2508.2)$ are notoriously absent from this table because they are recovered in very low yield, causing their methylated counterparts to become undetectable.
Overall, the set in Table 2 represents a $\sim 51 \%$ sequence coverage of the full-length protein.

In principle, due to the aforementioned random (Poissonlike) character of the diazirine modification reaction, the EM metric should depend linearly on the size of the polypeptide. This general trend is observed in Table 2 (e.g., pentadeca-

Table 2. MALDI-TOF Mass Analysis of Tryptic Peptides Derived from Lysozyme Labeled Under Native or Denaturing Conditions

\begin{tabular}{|c|c|c|c|c|c|}
\hline \multirow[t]{2}{*}{ Peptide sequence $^{\mathrm{a}}$} & \multirow{2}{*}{$\begin{array}{l}m / z \text { (Monoisotopic) } \\
\text { measured for }[\mathrm{M}+\mathrm{H}]^{+}\end{array}$} & \multirow{2}{*}{$\begin{array}{l}m / z \text { (Monoisotopic) measured } \\
\text { for }\left[\mathrm{M}+\mathrm{H}+\mathrm{CH}_{2}\right]^{+}\end{array}$} & \multicolumn{3}{|c|}{ EM\% (mol : $\mathrm{CH}_{2} /$ mol peptide $)^{\mathrm{b}}$} \\
\hline & & & $\mathrm{N}$ & U & $\mathrm{U} / \mathrm{N}^{\mathrm{c}}$ \\
\hline $\mathrm{H}_{15} \mathrm{GLDNYR}_{21}$ & 874.4 & 888.4 & 2.0 & 2.3 & 1.13 \\
\hline $\mathrm{F}_{34}$ ESNFNTQATNR $_{45}$ & 1428.6 & 1442.6 & 8.3 & 3.6 & 0.43 \\
\hline $\mathrm{N}_{46}$ TDGSTDYGILQINSR $_{61}$ & 1753.8 & 1767.8 & 2.5 & 2.7 & 1.11 \\
\hline $\mathrm{W}_{62} \mathrm{WCNDGR}_{68}$ & $993.4^{\mathrm{d}}$ & $1007.4^{\mathrm{d}}$ & 6.1 & 3.6 & 0.58 \\
\hline $\mathrm{I}_{98}$ VSDGNGMNAWVAWR $_{112}$ & $1675.8(1676.8)^{\mathrm{e}}$ & $1689.8(1690.8)^{\mathrm{e}}$ & 26.7 & 16.8 & 0.63 \\
\hline $\mathrm{G}_{117} \mathrm{TDVQAWIR}_{125}$ & 1045.5 & 1059.5 & 5.3 & 8.5 & 1.56 \\
\hline
\end{tabular}

Lysozyme samples $(1 \mathrm{mg} / \mathrm{mL})$ were labeled in the native $(\mathrm{N})$ or unfolded $(\mathrm{U}, 8 \mathrm{M}$ urea) states. The reaction conditions for modification were as follows: diazirine inflow rate of $1.0 \mathrm{~mL} / \mathrm{min}$, input plus photolysis phase lasting $40 \mathrm{~min}$, steady-state diazirine concentration $\left(\mathrm{C}_{0}\right) \sim 0.9$ AU. After reduction, carbamido methylation and digestion with trypsin, modified samples of lysozyme were analyzed by MALDI-MS. For details see Materials and Methods.

${ }^{a}$ Unambiguous identification of each peptide was achieved by MS/MS after MALDI-MS or ESI-MS analyses.

${ }^{\mathrm{b}}$ The extent of modification (EM\%) is expressed as mol of $\mathrm{CH}_{2}$ incorporated per $100 \mathrm{~mol}$ of protein, normalized by the load of diazirine reagent. A typical data set is shown. For all peptides, only the unlabeled and monomethylated species exist in the mixture (see Figure 4 and main text). Thus, Equation 6 becomes $E M \% \approx 100 \cdot I_{1} /\left(I_{0}+I_{1}\right)$. Numbers in the table represent calculations run on the monoisotopic species. However, if instead the full envelope of isotopic species is used, similar results are obtained (data not shown).

${ }^{\mathrm{c}}$ The labeling U/N ratio is calculated as the extent of methylene labeling (as estimated by the EM\%) of a sample modified under denaturing conditions (U) relative to a sample modified in the native state $(\mathrm{N})$.

${ }^{\mathrm{d}}$ The $\mathrm{m} / \mathrm{z}$ values shown correspond to the carbamido methylated species.

${ }^{\mathrm{e}}$ Values correspond to the original and deamidated species (between parentheses). EM\% calculated with the latter yield comparable values (data not shown). 
peptide 98-112 versus heptapeptide 15-21), but a careful analysis should necessarily include the influence of dissimilar peptide environments in each conformational state. In an attempt to rationalize the EM values found in the context of the tertiary structure of the target protein, we present Figure 5. Here, we describe the environment around each amino acid site by (1 estimating local solvent accessibility (SASA) along the lysozyme sequence, a measure derived from known crystallographic structures of this protein, and (2) plotting a classical Kyte and Doolittle hydrophobicity profile [53]. It becomes immediately obvious that peptide 46-61 (shown in grey) maps to a very hydrophobic core region with minimal solvent exposure. Thus, one can readily explain why, despite its high molecular weight, its measured EM value results anomalously low, e.g., by comparison with peptide 98-112, of similar length, but showing moderate solvent exposure and located in a predominantly polar milieu. On the other hand, a cross-comparison between states $\mathrm{N}$ and $\mathrm{U}$ is also possible, where the same site is able to be probed by diazirine in two radically different conformations. Here, the ratio $\mathrm{U} / \mathrm{N}$ becomes a useful signal independent of peptide molecular weight because it is able to sense changes due to the different local chemical environment and/or the distinctive dynamics of the polypeptide. As illustrated in Table 2, U/N ratios do not often adopt values larger than one, as would be expected from a generalized increase in SASA. In fact, it is even possible to detect 'reverse' changes, due to singular aspects of local protein environments. These include (1) the well-known

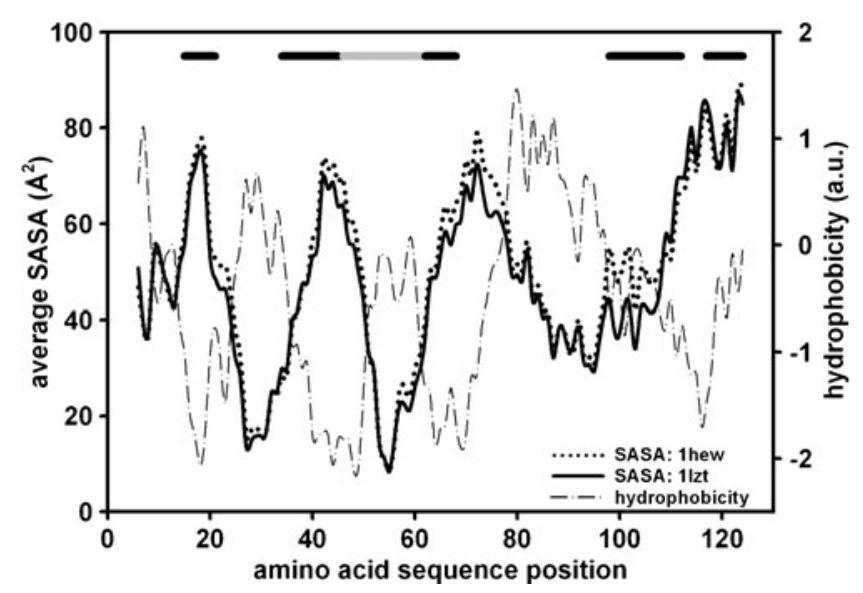

Figure 5. Local solvent exposure and hydrophobicity of lysozyme. The solvent accessible surface area (SASA) was calculated with SurfaceRacer [52] using van der Waals atomic radii according to the parameter set defined by Richards [5] and a probe radius of $1.4 \AA$ on pdb structures 1hew and 1lzt. Individual amino acid accessibility values were averaged along the sequence over a moving undecapeptide window. The hydrophobicity plot uses the Kyte and Doolittle scale [53] and an averaging window of the same size. The grey horizontal bar indicates the location of peptide 46-61 and the remaining peptides are shown with black bars (see Table 2) existence of cavities and crevices, locations that would preferentially lodge the diazirine reagent, and/or (2) the stabilization of partially folded forms, endowed with higher flexibility, enclosing hydrophobic spaces accessible to the aqueous solvent or the reagent. This observation was amply documented in previous studies of our laboratory, where peptide maps of $\alpha$-lactalbumin and $\beta$-lactamase (B. licheniformis $\beta$-lactamase) were carried out after photolabeling with ${ }^{3} \mathrm{H}$-diazirine $[33,36,37]$. Indeed, lysozyme does not depart from this rule, since plenty of evidence exists on the persistence of residual structure even in the presence of $8 \mathrm{M}$ urea $[54,55]$. Under denaturing conditions, significant deviations from random coil behavior occur. This outcome arises from weak clustering of hydrophobic groups near tryptophan residues and/or the presence of sequence-dependent long-range interactions, giving rise to a collection of compact, somewhat less mobile conformers stabilized by the network of disulfide bridges. Therefore, a naïve interpretation predicting uniform solvent exposure for peptides derived from the $U$ ensemble is far from warranted. Moreover, $\mathrm{U} / \mathrm{N}$ values close to unity are also expected for highly exposed, flexible entities in state $\mathrm{N}$, such as peptide 15-21, which would experience little or no change upon protein denaturation. At the other extreme, highly structured regions, e.g., including closed-packed volumes or cross-links in the native state (peptide 46-61), might result difficult to disorganize, therefore also rendering $\mathrm{U} / \mathrm{N}$ values close to unity.

Altogether, this new level of analysis demonstrates the ability of the technique to dissect an overall conformational change, such as that illustrated in Table 1, into useful local structural information.

\section{Sites of Labeling Can Be Pinpointed to Single Amino Acid Residues}

Peptide $\mathrm{F}_{34} \mathrm{ESNFNTQATNR}_{45}$ was selected to illustrate the fact that the identification of methylated sites can indeed be extended to the amino acid level. The basis for this analysis lies in the comparison of the fragmentation profiles after CID-MS/MS of the monomethylated species $(\mathrm{m} / \mathrm{z}$ 1442.6, Figure 6a) with that corresponding to the parent (unlabeled) peptide $(\mathrm{m} / \mathrm{z}$ 1428.6, Figure $6 \mathrm{~b})$. (The same series of fragments were observed after MALDI MS/MS and post source decay (PSD) fragmentation, results not shown). A closer check of the fragmentation pattern shown in Figure 6a reveals that in many instances, the fragment ion is accompanied by a methylated counterpart (marked with the index $\mathrm{m}$ ). In both spectra, the y series of fragments (monocharged peaks $y_{3}$ to $y_{10}$ ) is readily apparent because of its higher yield. Most significantly, there is also evidence of the presence of methylated species in the b-ion series. Within this set, as a result of consecutive cleavage reactions, it is generally observed that lower b-ions occur with disproportionate abundance [56]. It is noteworthy to point out that the fragmentation pattern observed shows the almost exclusive presence of the methylated b-ions $\left(b_{2} \mathrm{~m}\right.$ to $\left.b_{6} \mathrm{~m}\right)$, and the $\mathrm{a}_{2} \mathrm{~m}$ 


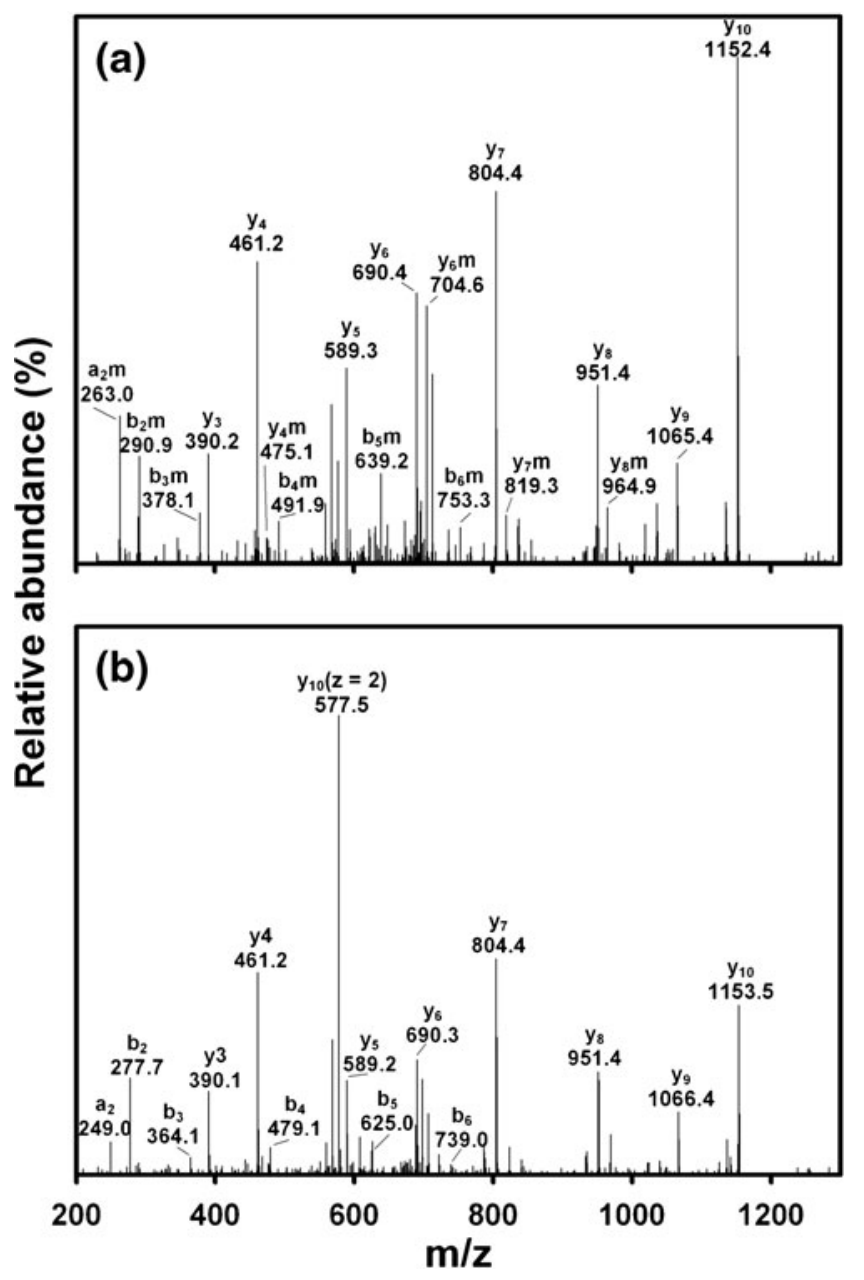

Figure 6. MS/MS spectra of peptide $\mathrm{F}_{34} \mathrm{ESNFNTQATNR}_{45}$ derived from lysozyme. The fragmentation patterns are shown, corresponding to the collection of monomethylated species $[\mathrm{m} / \mathrm{z} 1442.6, \mathbf{a})]$ and the unlabeled counterpart $[\mathrm{m} / \mathrm{z}$ 1428.6, (b)]. Entities identified as methylated products are named after their precursors and marked with the index $\mathrm{m}$. Each spectrum represents the average of two scans

species derived from $\mathrm{b}_{2} \mathrm{~m}$ by loss of the carbonyl moiety. Similarly, a comparable recovery of the unmodified $a$ and $b$ fragment series is observed in Figure 6b. Taken together, these results point to the occurrence of methylated spots definitely associated to amino acids F34 or E35, and to an additional, more diffuse site of modification located between amino acids 42 and 45 . Thus, although in this case a detectable bias exists toward labeling one end of this peptide, the distributed character of the labeling observed points to the general lack of chemical selectivity characteristic of the diazirine reagent.

In principle, from a global analysis of relative peak intensities corresponding to each unmodified peak and its methylated counterpart, it is possible to calculate EM values from which the probabilities of reaction at individual (amino acid) sites can be derived. As a first approximation to this problem, one attempt to interpret the available results considers fitting a model where the observed probability of reaction of a given fragment results from the sum of independent probabilities at each site. Current efforts in our laboratory focus on gathering enough data to properly satisfy statistical criteria and evaluate this possibility in a meaningful way. One should bear in mind that even though methylation might conceivably represent one minimal possible modification of the polypeptide, its relative effect on the ability of the ion to fly will necessarily increase as the fragment becomes shorter. Therefore, although care should be exercised to interpret results in a strict quantitative fashion, the ability to accurately pinpoint the site of methylation at the amino acid level appears as a feasible goal in the short term.

As we were in the process of revising the present manuscript, a carbene labeling approach was published based on the amino acid 'photoleucine' aimed at analyzing protein topography [57]. 'Photoleucine' is a large, chiral, zwitterionic, water-soluble molecule. Thus, its ability to access the protein surface will not only depend on its geometry (size and shape), but will also be compounded with other issues, such as charge, amphipatic character, and orientation effects. These are key factors determining the distribution of labeled sites. By contrast, the small diazirine molecule serves as a useful mimic of the water solvent, therefore being able to finely probe molecular details. Added advantages of diazirine will be the expected minimal or null orientation effects on the addition reaction and the obvious lack of internal conversion reactions. It remains to be seen if other larger and more complex entities could adequately sample SASA as the primary phenomenon.

In regard to the reliable quantification of products derived from 'photoleucine', the underlying assumption is that ionization efficiency will not be affected by the modification. Strictly, this might never hold true, but by comparison, methylation (as a result of the reaction with diazirine) will represent a lesser influence than the addition of a full amino acid. On the other hand, in the 'photoleucine' approach, protein fragmentation and unambiguous identification of labeled peptides followed by quantitative estimation of labeling yields at individual sites of reaction remains an open issue.

\section{Conclusions}

Results presented herein demonstrate the worth of the convergence of a photochemical method able to probe the same surface as water in protein molecules with the added advantage provided by MS for detecting modified products at superior resolution. In this fashion, diazirine labeling coupled to MS is uniquely suited to shed light on conformational features of the native as well as non-native states, the latter not easily addressable by other methods. The added dimension provided by the fragmentation of the polypeptide chain allows one to locate the covalent tag along the amino acid sequence, therefore enabling the mapping of solvent accessibility at different sites. Overall, the observations 
herein presented demonstrate the practicability of attaining small peptide or even amino acid resolution in defining those target sites reached by the diazirine reagent. Further developments include the feasibility of automating the procedure to apply it in high-throughput proteomic and/or interactomic efforts.

\section{Acknowledgments}

For the interpretation of spectra, the expert advice of Ms. Susana Linskens and Mr. Carlos Paván (LANAIS-PRO, UBA-CONICET) is kindly acknowledged. G.E.G. was supported by a fellowship from Fundación YPF. This research was supported by grants to JMD from UBACyT, CONICET, and ANPCyT.

\section{References}

1. Hammes, G.G.: Spectroscopy for the biological sciences. John Wiley and Sons, Inc., New Jersey (2005)

2. Fersht, A.: Structure and Mechanism in Protein Science: A Guide to Enzyme Catalysis and Protein Folding. W. H. Freeman and Company, New York (1999)

3. Creighton, T.E. (ed.): Protein Folding. W. H. Freeman and Company, New York (1992)

4. Pain, R.H. (ed.): Mechanisms of Protein Folding. IRL Press at Oxford University Press, Oxford, UK (1994)

5. Richards, F.M.: Areas, Volumes, Packing, and Protein Structure. Annu. Rev. Biophys. Bioeng. 6, 151-176 (1977)

6. Richards, F.M., Lamed, R., Wynn, R., Patel, D., Olack, G.: Methylene as a Possible Universal Footprinting Reagent that will Include Hydrophobic Surface Areas. Overview and Feasibility: Properties of Diazirine as a Precursor. Protein Sci. 9, 2506-2517 (2000)

7. Makhatadze, G.I., Privalov, P.L.: Heat Capacity of Proteins. I. Partial Molar Heat Capacity of Individual Amino Acid Residues in Aqueous Solution: Hydration Effect. J. Mol. Biol. 213, 375-384 (1990)

8. Privalov, P.L., Makhatadze, G.I.: Heat Capacity of Proteins. II. Partial Molar Heat Capacity of the Unfolded Polypeptide Chain of Proteins: Protein Unfolding Effects. J. Mol. Biol. 213, 385-291 (1990)

9. Livingstone, J.R., Spolar, R.S., Record Jr., M.T.: Contribution to the Thermodynamics of Protein Folding from the Reduction in Water-Accessible Nonpolar Surface Area. Biochemistry 30, 4237-4244 (1991)

10. Prabhu, N.V., Sharp, K.A.: Heat Capacity in Proteins. Annu. Rev. Phys. Chem. 56, 521-548 (2005)

11. Myers, J.K., Pace, C.N., Scholtz, J.M.: Denaturant $m$ Values and Heat Capacity Changes: Relation to Changes in Accessible Surface Areas of Protein Unfolding. Protein Sci. 4, 2138-2148 (1995)

12. Geierhaas, C.D., Nickson, A.A., Lindorff-Larsen, K., Clarke, J., Vendruscolo, M.: BPPred: A Web-Based Computational Tool for Predicting Biophysical Parameters of Proteins. Protein Sci. 16, 125134 (2007)

13. Mendoza, V.L., Vachet, R.W.: Probing Protein Structure by Amino Acid-Specific Covalent Labeling and Mass Spectrometry. Mass Spectrom Rev. 28, 785-815 (2009)

14. Fitzgerald, M.C., West, G.M.: Painting Proteins with Covalent Labels: What's in the Picture? J. Am. Soc. Mass Spectrom. 20, 1193-1206 (2009)

15. Englander, S.W.: Hydrogen Exchange and Mass Spectrometry: A Historical Perspective. J. Am. Soc. Mass Spectrom. 17, 1481-1489 (2006)

16. Englander, S.W., Mayne, L., Krishna, M.M.: Protein Folding and Misfolding: Mechanism and Principles. Q Rev. Biophys. 40, 287-326 (2007)

17. Zhu, M.M., Rempel, D.L., Du, Z., Gross, M.L.: Quantification of Protein-Ligand Interactions by Mass Spectrometry, Titration, and H/D Exchange: PLIMSTEX. J. Am. Chem. Soc. 125, 5252-5253 (2003)

18. Tullius, T.D., Dombroski, B.A.: Iron (II) EDTA Used to Measure the Helical Twist Along Any DNA Molecule. Science 230, 679-681 (1985)
19. Tullius, T.D., Dombroski, B.A., Churchill, M.E.A., Kam, L.: Hydroxyl Radical Footprinting: A High Resolution Method for Mapping ProteinDNA Contacts. In: Wu, R., Grossman, L., Moldave, K. (eds.) Recombinant DNA Methodology. Academic Press, San Diego, CA (1989)

20. Jain, S.S., Tullius, T.D.: Footprinting Protein-DNA Complexes Using the Hydroxyl Radical. Nat. Protoc. 3, 1092-1100 (2008)

21. Ermacora, M.R., Delfino, J.M., Cuenoud, B., Schepartz, A., Fox, R.O.: Conformation-Dependent Cleavage of Staphylococcal Nuclease with a Disulfide-Linked Iron Chelate. Proc. Natl. Acad. Sci. U.S.A. 89, 63836387 (1992)

22. Ermacora, M.R., Ledman, D.W., Hellinga, H.W., Hsu, G.W., Fox, R. O.: Mapping Staphylococcal Nuclease Conformation Using an EDTAFe Derivative Attached to Genetically Engineered Cysteine Residues. Biochemistry 33, 13625-13641 (1994)

23. Maleknia, S.D., Brenowitz, M., Chance, M.R.: Millisecond Radiolytic Modification of Peptides by Synchrotron X-Rays Identified by Mass Spectrometry. Anal. Chem. 71, 3965-3973 (1999)

24. Maleknia, S.D., Ralston, C.Y., Brenowitz, M.D., Downard, K.M., Chance, M.R.: Determination of Macromolecular Folding and Structure by Synchrotron X-Ray Radiolysis Techniques. Anal. Biochem. 289, 103-115 (2001)

25. Maleknia, S.D., Downard, K.M.: Unfolding of Apomyoglobin Helices by Synchrotron Radiolysis and Mass Spectrometry. Eur. J. Biochem. 268, 5578-5588 (2001)

26. Takamoto, K., Chance, M.R.: Radiolytic Protein Footprinting with Mass Spectrometry to Probe the Structure of Macromolecular Complexes. Annu. Rev. Biophys. Biomol. Struct. 35, 251-276 (2006)

27. Nukuna, B.N., Goshe, M.B., Anderson, V.E.: Sites of Hydroxyl Radical Reaction with Amino Acids Identified by (2)H NMR Detection of Induced (1)H/(2)H Exchange. J. Am. Chem. Soc. 123, 1208-1214 (2001)

28. Gau, B.C., Sharp, J.S., Rempel, D.L., Gross, M.L.: Fast Photochemical Oxidation of Protein Footprints Faster than Protein Unfolding. Anal. Chem. 81, 6563-6571 (2009)

29. Bayley, H.: Photogenerated Reagents in Biochemistry and Molecular Biology. Elsevier, Amsterdam (1983)

30. Scriven, E.F.V. (ed.): Azides and Nitrenes. Reactivity and Utility. Academic Press, Orlando (1984)

31. Brunner, J.: New Photolabeling and Crosslinking Methods. Annu. Rev. Biochem. 62, 483-514 (1993)

32. Delfino, J.M., Schreiber, S.L., Richards, F.M.: Design, Synthesis, and Properties of a Photoactivatable Membrane-Spanning Phospholipidic Probe. J. Am. Chem. Soc. 115, 3458-3474 (1993)

33. Craig, P.O., Ureta, D.B., Delfino, J.M.: Probing Protein Conformation with a Minimal Photochemical Reagent. Protein Sci. 11, 1353-1366 (2002)

34. Frey, H.M.: The photolysis of Diazirines. Adv. Photochem. 4, 225-256 (1966)

35. Turro, N.J., Cha, Y., Gould, I.R.: Reactivity and Intersystem Crossing of Singlet Methylene in Solution. J. Am. Chem. Soc. 109, 2101-2107 (1987)

36. Craig, P.O., Gómez, G.E., Ureta, D.B., Caramelo, J.J., Delfino, J.M.: Experimentally Approaching the Solvent-Accessible Surface Area of a Protein: Insights into the Acid Molten Globule of Bovine $\alpha$-Lactalbumin. $J$. Mol. Biol. 394, 982-993 (2009)

37. Ureta, D.B., Craig, P.O., Gómez, G.E., Delfino, J.M.: Assessing Native and Non-Native Conformational States of a Protein by Methylene Carbene Labeling: The Case of Bacillus licheniformis $\beta$-lactamase. Biochemistry 46, 14567-14577 (2007)

38. Gómez, G.E., Cauerhff, A., Craig, P.O., Goldbaum, F.A., Delfino, J.M.: Exploring Protein Interfaces with a General Photochemical Reagent. Protein Sci. 15, 744-752 (2006)

39. Nuss, J.E., Alter, G.M.: Denaturation of Replication Protein A Reveals an Alternative Conformation with Intact Domain Structure and Oligonucleotide Binding Activity. Protein Sci. 13, 1365-1378 (2004)

40. Chance, M., Ed. Mass Spectrometry Analysis for Protein-Protein Interactions and Dynamics; John Wiley and Sons, Inc.: NJ, 2008;

41. Kaltashov, I.A., Eyles, S.J.: Eds. Mass Spectrometry in Biophysics. Conformation and Dynamics of Biomolecules; John Wiley and Sons, Inc., NJ (2005)

42. Ibarra-Molero, B., Loladze, V.V., Makhatadze, G.I., Sanchez-Ruiz, J.M.: Thermal Versus Guanidine-Induced Unfolding of Ubiquitin. An Analysis in Terms of the Contributions from Charge-Charge Interactions to Protein Stability. Biochemistry 38, 8138-8149 (1999) 
43. Kronman, M.J., Andreotti, R.E.: Inter- and intramolecular interactions of $\mapsto$-lactalbumin. I. The apparent heterogeneity at acid pH. Biochemistry 3, 1145-1151 (1964)

44. Canfield, R.E.: Peptides Derived from Tryptic Digestion of Egg White Lysozyme. J. Biol. Chem. 238, 2691-2697 (1963)

45. Landon, M., Evans, W.H., Smith, E.L.: Subtilisin Carlsberg. 3. Isolation and Amino Acid Composition of Chymotryptic Peptides. J. Biol. Chem. 243, 2165-2171 (1968)

46. Ellerby, L.M., Escobar, W.A., Fink, A.L., Mitchinson, C., Wells, J.A.: The Role of Lysine-234 in $\beta$-Lactamase Catalysis Probed by SiteDirected Mutagenesis. Biochemistry 29, 5797-5806 (1990)

47. Davie, E.W., Neurath, H.: The Terminal Groups of the Soy bean trypsin inhibitor. J. Biol. Chem. 212, 507-514 (1955)

48. Webster, G.C.: Comparison of Direct Spectrophotometric Methods for the Measurement of Protein Concentration. Biochim. Biophys. Acta 207, 371-373 (1970)

49. Crouch, T.H., Klee, C.B.: Positive Cooperative Binding of Calcium to Bovine Brain Calmodulin. Biochemistry 19, 3692-3698 (1980)

50. Ohme, R., Schmitz, E.: Notiz über eine einfache synthese des CycloDiazomethans. Chem. Ber. 97, 297-298 (1964)

51. Waxdal, M.J., Konigsberg, W.H., Henley, W.L., Edelman, G.M.: The Covalent Structure of a Human $\gamma$ G-Immunoglobulin: II. Isolation and
Characterization of the Cyanogen Bromide Fragments. Biochemistry 7, 1959-1966 (1968)

52. Tsodikov, O.V., Record Jr., M.T., Sergeev, Y.V.: A Novel Computer Program for Fast Exact Calculation of Accessible and Molecular Surface Areas and Average Surface Curvature. J. Comput. Chem. 23, 600-609 (2002)

53. Kyte, J., Doolittle, R.F.: A Simple Method for Displaying the Hydropathic Character of a Protein. J. Mol. Biol. 157, 105-132 (1982)

54. Schwalbe, H., Fiebig, K.M., Buck, M., Jones, J.A., Grimshaw, S.B., Spencer, A., Glaser, S.J., Smith, L.J., Dobson, C.M.: Structural and Dynamic Properties of a Denatured Protein. Heteronuclear 3D NMR Experiments and Theoretical Simulations of Lysozyme in $8 \mathrm{M}$ Urea. Biochemistry 36, 8977-8991 (1997)

55. Wirmer, J., Berk, H., Ugolini, R., Redfield, C., Schwalbe, H.: Characterization of the Unfolded State of Bovine $\mapsto$-Lactalbumin and Comparison with Unfolded States of Homologous Proteins. Protein Sci. 15, 1397-1407 (2006)

56. Kinter, M., Sherman, N.E.: Protein Sequencing and Identification Using Tandem Mass Spectrometry, p. 73. Wiley Interscience, New York, NY (2000)

57. Jumper, C.C., Schriemer, D.C.: Mass Spectrometry of Laser-Initiated Carbene Reactions for Protein Topographic Analysis. Anal Chem. 83, 2913-2920 (2011) 